\title{
Atraindo Meninas para a Ciência da Computação: Métodos e Ferramentas
}

\author{
Josilene Aires Moreira ${ }^{1}$, Giorgia de Oliveira Mattos ${ }^{1}$, Livia de Sousa Barreto ${ }^{1}$, \\ Isabela Nascimento Cavaco ${ }^{1}$, Ricardo Moreira da Silva ${ }^{2}$ \\ ${ }^{1}$ Centro de Informática - Universidade Federal da Paraíba (UFPB) \\ Avenida dos Escoteiros $-\mathrm{s} / \mathrm{n}^{\circ}$ Mangabeira VII - 58.055-000 \\ João Pessoa - PB - Brazil \\ ${ }^{2}$ Departamento de Engenharia de Produção - Universidade Federal da Paraíba (UFPB) \\ Jardim Cidade Universitária - 58.051-001 - João Pessoa - PB - Brazil.

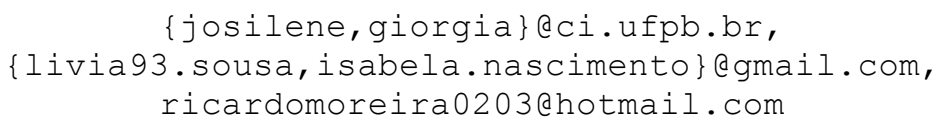

\begin{abstract}
Women are under-represented in Computer Science undergraduate programs and careers. In order to encourage girls to join this area, several initiatives are being conducted worldwide. This article examines case studies applied in high school and elementary, reporting types of activities, duration, number of students, evaluation processes, separation of groups by gender and tools used, among others. We found that the production-based learning (programming, modeling, design, prototyping and assembly) was the most used approach, and Alice the most widely used tool. Most initiatives lasted a week, were attended by 30 students on average and were performed only with girls.
\end{abstract}

Resumo. As mulheres são minoria nos cursos e carreiras de Ciência da Computação. A fim de incentivar meninas a ingressarem nesta área, diversas iniciativas estão sendo conduzidas em todo o mundo. Este artigo analisa casos de sucesso aplicados no Ensino Médio e Fundamental, relatando os tipos de atividades, duração, número de estudantes, processos de avaliação, separação de grupos por gênero e ferramentas utilizadas, entre outros. Verificamos que o aprendizado baseado em produção (programação, modelagem, design, prototipação e montagem) foi a abordagem mais utilizada, e Alice a ferramenta mais usada. A maioria das iniciativas durou uma semana, contou com 30 alunas e foi realizada apenas com meninas.

\section{Introdução}

Existe uma lacuna entre os gêneros no que diz respeito ao uso de computadores, como atesta o relatório sobre as habilidades de pessoas adultas realizado pela OECD Organização para a Cooperação e Desenvolvimento Econômico [Oecd 2013]. Com relação ao uso de ferramentas computacionais, os homens obtiveram melhor desempenho em todos os países investigados; adicionalmente, superaram as mulheres na habilidade "resolução de problemas" em mais da metade dos países e economias. O Brasil encontra-se entre os países que apresentaram as maiores diferenças entre homens 
V Congresso Brasileiro de Informática na Educação (CBIE 2016)

Anais dos Workshops do V Congresso Brasileiro de Informática na Educação (CBIE 2016)

e mulheres: eles obtiveram em média vinte pontos a mais do que elas neste item da avaliação, juntamente com a Colômbia, a China e a Eslováquia. A diferença nos demais países avaliados ficou em sete pontos em média.

$\mathrm{Na}$ área de Ciência da Computação as mulheres são minoria nos cursos superiores e nas pós-graduações em todo o mundo. Na União Européia, a proporção de mulheres com doutorado em Ciência da Computação é de apenas $24 \%$. O Instituto Europeu de Informática, o qual relata dados sobre o ingresso de estudantes por gênero em Informática e Computação, afirma que na Holanda as mulheres representaram apenas $13,8 \%$ do total de matrículas em 2013; na Suíça, 10,3\% e 17\% no Reino Unido [Pereira and Meyer 2013]. Nos Estados Unidos o número de mulheres matriculadas nos cursos de Bacharelado em Ciência da Computação e Informática é menor do que $20 \%$ [Jung and Apedoe 2013], enquanto no Brasil a proporção é de menos de 17\% [Inep 2013].

A Paraíba acompanha a tendência nacional apresentando uma proporção de mulheres ainda menor. Os cursos de Bacharelado em Ciência da Computação e Engenharia da Computação do Campus I da UFPB em João Pessoa, Paraíba, apresentam uma forte predominância masculina, conforme mostrado na Tabela 1 [Nti 2015]. Apenas 13\% de mulheres estão matriculadas nos cursos de Bacharelado e Engenharia da Computação, contra $87 \%$ de homens. O Curso de Matemática Computacional, iniciado no ano de 2013 , atraiu inicialmente uma fatia um pouco maior de mulheres, que representam $21 \%$ do total de estudantes. Já no curso de Licenciatura em Computação, oferecido na modalidade a distância, os homens são $69 \%$ do total de alunos, mesmo sendo a licenciatura um curso tipicamente feminino.

Tabela 1. Estudantes matriculados nos cursos da área de Computação da UFPB - Período 2014.1

\begin{tabular}{|c|c|c|c|c|c|}
\hline \multirow{2}{*}{ Cursos } & \multicolumn{2}{|c|}{ Alunos } & \multicolumn{2}{|c|}{ Alunas } & \multirow{2}{*}{$\begin{array}{l}\text { Total } \\
\text { Num }\end{array}$} \\
\hline & Num & $\%$ & Num & $\%$ & \\
\hline Bacharelado em Ciência da Computação & 314 & 87 & 45 & 13 & 359 \\
\hline Engenharia da Computação & 208 & 87 & 31 & 13 & 239 \\
\hline Matemática Computacional & 110 & 79 & 30 & 21 & 140 \\
\hline Licenciatura em Computação & 259 & 69 & 119 & 31 & 378 \\
\hline Total & 891 & 80 & 225 & 20 & 1116 \\
\hline
\end{tabular}

Este artigo apresenta iniciativas adotadas por outros países que também buscam alterar a realidade da baixa participação feminina na Ciência da Computação através de atividades junto a estudantes do Ensino Médio e Fundamental. Foram focados os casos de sucesso, identificando métodos, ferramentas, público-alvo, participação e estratégias, entre outros, que possam promover uma melhor compreensão da problemática e fomentar a adoção de práticas efetivas para a atração das alunas para esta área do conhecimento.

\section{A Inclusão Feminina na Ciência e Tecnologia}

A igualdade de gênero é um dos dezessete Objetivos de Desenvolvimento Sustentável (ODS) estabelecidos pela Organização das Nações Unidas (ONU) com mais de 150 líderes mundiais para combater diversos males da sociedade, compromisso que foi 
firmado em 2015 para ser alcançado até 2030. A igualdade de gênero visa promover o empoderamento feminino e é considerada importante tanto no aspecto econômico e social como para atingir outras metas prioritárias, em especial aquelas ligadas à pobreza, fome, saúde e educação [Pnud 2015]. Na realidade, ainda é marcante a permanência das mulheres em campos do conhecimento tradicionalmente ligados à identidade feminina, como Psicologia, Linguística, Nutrição, Serviço Social, Fonoaudiologia, Economia Doméstica, Enfermagem, que remetem aos "papéis" de gênero ligados à doação, ao cuidado e à maternidade. Áreas do conhecimento como Astronomia, Matemática, Engenharias, Ciência da Computação e, sobretudo, Física constituem as áreas de menor participação das mulheres [Olinto 2011]. Adicionalmente, em 2009, comparando-se a média anual dos rendimentos dos homens e das mulheres no Brasil, verificou-se que, em média, as mulheres ganham em torno de $72,3 \%$ do rendimento recebido pelos homens. Na Europa, embora essa diferença seja inferior à verificada no Brasil, pois elas ganham em torno de $85 \%$ do rendimento masculino, a taxa vem se mantendo estável desde 2003 [Pnud 2015].

Nesse contexto o currículo escolar exerce papel fundamental uma vez que, não apenas está envolvido com a transmissão de fatos e conhecimentos objetivos, mas antes envolve a construção de significados e valores culturais. E estes valores culturais estão estreitamente ligados às relações sociais de poder e de desigualdade. Em seu livro intitulado "A dominação masculina", Bordieu [Bourdieu 2002] destaca que, tradicionalmente, os homens detêm o monopólio da criação e utilização das máquinas e objetos eletrônicos. Por outro lado, enfatiza que a dominação masculina não se impõe mais como indiscutível, e que o mais importante de todos os fatores de mudança capazes de transformar esta visão é a atuação da instituição escolar sobre o papel dos gêneros.

Este artigo analisa detalhadamente um conjunto de estratégias que vem sendo utilizadas principalmente nos Estados Unidos para promover a inclusão feminina na área de Ciência da Computação. O principal objetivo é identificar e mapear iniciativas, ferramentas e métodos que apresentaram bons resultados - casos de sucesso - e que possam ser utilizados também no Brasil, a fim de contribuir para a diminuição das desigualdades nos cursos e carreiras desta área o que, a médio e longo prazo, contribuirá para a inserção feminina em um mercado com maior valor social e maior renda.

\section{Metodologia}

A pesquisa foi realizada através da busca de artigos científicos nos portais da ACM, IEEE e Science Direct em outubro de 2015, usando a string de busca "Girls in Computer Science", limitando os resultados ao período de 2010 a 2015. A busca foi refinada para incluir termos como "High school", "K-12" e "Middle school", quando então os artigos foram selecionados manualmente através da leitura dos resumos. Nesta etapa, diversos artigos foram descartados por não apresentarem os métodos, ferramentas e técnicas, que seriam o principal foco. Restaram então 23 artigos que foram classificados, através da leitura, como de Alta, Média e Baixa relevância para o tema. Vinte deles apresentaram estudos sobre iniciativas conduzidas nos Estados Unidos, dois na Arábia Saudita e um na Alemanha. A partir deste conjunto de artigos, foi realizado um mapeamento detalhado das técnicas, estratégias, metodologias e ferramentas utilizadas, as quais serão apresentadas a seguir. As abordagens são apresentadas de 
V Congresso Brasileiro de Informática na Educação (CBIE 2016)

Anais dos Workshops do V Congresso Brasileiro de Informática na Educação (CBIE 2016)

acordo com a etapa de ensino a qual foram dirigidas, incluindo o ensino fundamental e o ensino médio.

\subsection{Ensino Fundamental}

As alunas do ensino fundamental foram o foco de onze trabalhos que buscaram, em geral, motivar as alunas desde cedo na grade curricular, para que desmistificassem e conhecessem melhor a área de Ciência da Computação. Para alguns, é importante que as meninas conheçam a área e comecem a identificar aspectos que mais gostam, como é afirmado por Hirsch [2013]:

Durante os anos intermediários do ensino fundamental ${ }_{1}$, as alunas começam a subestimar as suas habilidades técnicas e a buscarem mais a popularidade entre os colegas do que um bom desempenho acadêmico. Já durante o ensino médio, elas se matriculam menos do que os meninos nas disciplinas de matemática e ciências, o que começa a provocar uma falta de conhecimento necessário para que ingressem em cursos superiores nas áreas de STEM.

As atividades foram planejadas de diversas maneiras, em sua maior parte como acampamentos de verão [Hirsch 2013] [Hulsey 2014] [Khoja 2012] [Starrett 2015], horário extra após aula [Denner 2012] [Stewart-Gardiner 2013], oficinas (workshops) [Webb 2011] [Robinson 2014] [Webb and Rosson 2013] e disciplinas ministradas durante as aulas tradicionais [Webb 2012]. Alguns optaram por trabalhar os grupos de ambos os gêneros, implementados em [Hirsch 2013][ Stewart-Gardiner 2013] e [Webb 2012], com destaque para [Hirsch 2013] que conduziu grupos diversos baseados em gênero (apenas meninas, misto e apenas meninos), para realizar um comparativo de aprendizado e comportamento entre eles. O restante [Hulsey 2014] [Khoja 2012] [Starrett 2015] [Denner 2012] [Webb 2011] [Robinson 2014] e [Webb and Rosson 2013] optou por dar atenção apenas às meninas, pois as mesmas se sentem mais à vontade para participar e socializar com as demais quando não há presença dos meninos nas atividades, como foi descoberto no estudo comparativo:

[...] Nos programas mistos as garotas tenderam a focar mais nas tarefas do que na interação com os outros alunos e alunas, enquanto que nas atividades realizadas em programas apenas com meninas elas foram capazes de permanecer focadas e também interagir com as demais alunas do grupo. Em grupos mistos os alunos parecem ter se distanciado dos outros em alguma espécie de isolamento [Hirsch 2013].

O autor observa que todos os estudantes, meninos e meninas, alcançaram um desempenho semelhante nas tarefas, embora as meninas tenham demonstrado preferência em desenvolver as atividades com colegas do mesmo sexo. Esse comportamento diferenciado quando elas trabalham entre si é uma das conclusões:

[...] Pesquisas consideráveis podem ser encontradas descrevendo os vários benefícios de educação de gênero único para meninas, sugerindo melhoras na confiança delas, tornando mais provável que façam perguntas e mantenham comportamentos sociais nestas circunstâncias, os quais tendem a desaparecer quando da dominância masculina na sala de aula [Hirsch 2013].

\footnotetext{
${ }^{1}$ No Brasil, corresponde ao período do $6^{\circ}$ ano até o $9^{\circ}$ do ensino fundamental, e nos Estados Unidos é chamado de Middle School, abrangendo tipicamente do $6^{\circ}$ até o $8^{\circ}$ ano.
} 
V Congresso Brasileiro de Informática na Educação (CBIE 2016)

Anais dos Workshops do V Congresso Brasileiro de Informática na Educação (CBIE 2016)

Com respeito às metodologias aplicadas pelos trabalhos, foram classificadas como mostrado na Tabela 2.

Tabela 2. Metodologias encontradas para o ensino fundamental

\begin{tabular}{|c|c|}
\hline Metodologia & Estudo \\
\hline Acampamentos e visitas a empresas & [Hirsch 2013] [Khoja 2012] [Starrett 2015] \\
\hline Grupos separados por gênero & {$[$ Hirsch 2013] } \\
\hline Atividades físicas & [Khoja 2012] \\
\hline $\begin{array}{c}\text { Aprendizado baseado em produção } \\
\text { (programação, modelagem, design, } \\
\text { prototipação e montagem) }\end{array}$ & $\begin{array}{c}\text { [Hulsey 2014] [Khoja 2012] [Starrett 2015] [Denner } \\
\text { 2012] [Webb 2011] [Murphy 2010] [Webb 2012], } \\
\text { [Robinson 2014] [Webb and Rosson 2013] }\end{array}$ \\
\hline $\begin{array}{c}\text { Aprendizado baseado em jogos } \\
\text { [Stewart-Gardiner 2013] }\end{array}$ & [Denner 2012] \\
\hline $\begin{array}{c}\text { Avaliação através de questionários } \\
\text { Avaliação através do conteúdo produzido }\end{array}$ & $\begin{array}{c}\text { [Hirsch 2013] [Hulsey 2014] [Khoja 2012] [Starrett } \\
\text { 2015] [Stewart-Gardiner 2013] [Webb 2012] }\end{array}$ \\
\hline Avaliação através de entrevistas & [Robinson 2014] [Webb and Rosson 2013] \\
\hline Contato direto com profissionais de sucesso & [Khoja 2012] [Webb and Rosson 2013] \\
\hline nárea
\end{tabular}

As atividades de aprendizagem foram $77,8 \%$ baseadas em produção (programação, modelagem, design, prototipação e montagem); $11,1 \%$ baseada na aplicação de jogos educativos, e outros $11,1 \%$ não relataram o tipo de atividade. Outras abordagens adotadas foram à realização de acampamentos e visitas a empresas locais [Hirsch 2013] [Khoja 2012][Starrett 2015], a adoção de um horário reservado para atividades físicas [Khoja 2012] e o contato direto das estudantes com mulheres profissionais [Khoja 2012] [Webb and Rosson 2013] para que pudessem enxergá-las como mulheres bem-sucedidas e fontes de inspiração, e para sanar dúvidas sobre o mercado de trabalho. O trabalho descrito em [Murphy 2010] usou exclusivamente a robótica. A prática de atividades físicas foi adotada em um acampamento de verão, e era praticada após o almoço, a fim de quebrar a rotina de estudos [Khoja 2012]. Como processo de avaliação das estudantes, $55,5 \%$ aplicaram questionários antes e após as atividades programadas, as demais usaram entrevistas.

\subsection{Ensino Médio}

As alunas do ensino médio foram público-alvo de sete trabalhos [Cohoon 2011] [Burge 2013] [Gannod 2014] [Al-Duwis 2013] [Rosson 2010] [Alhumoud 2014] e [Wellington 2010]. As atividades foram planejadas como acampamentos de verão [Burge 2013] [Gannod 2014] [Alhumoud 2014] e oficinas [Cohoon 2011] [Al-Duwis 2013] [Rosson 2010]. A maioria trabalhou com grupos exclusivamente de meninas [Burge 2013] [Gannod 2014] [Al-Duwis 2013] [Rosson 2010] [Alhumoud 2014] [Wellington 2010], e um deles com professores buscando conscientizá-las sobre o respeito das diferenças entre os gêneros existentes na área da computação, como afirmado em [Cohoon 2011]: 
V Congresso Brasileiro de Informática na Educação (CBIE 2016)

Anais dos Workshops do V Congresso Brasileiro de Informática na Educação (CBIE 2016)

Professores do Ensino Médio não se encontram bem preparados para promover o aumento da participação de alunas e de minorias de estudantes nas carreiras de Ciência da Computação. Por isso é importante prepará-los e, dessa forma, alcançar o resultado desejado de incrementar o número de alunas que irão buscar cursos superiores nesta área [Cohoon 2011].

As atividades de aprendizagem foram $71,2 \%$ baseadas em produção, através de programação, modelagem, design, prototipação e montagem; $14,4 \%$ referente à podcasts $^{2}$ criados por alunas de graduação em Ciência da Computação para mostrar conteúdos explorados por um cientista da computação e ao mesmo tempo apresentar um modelo feminino presente na área. Os 14,4\% são referentes ao trabalho [Cohoon 2011] realizado com professores e o mesmo não teve aplicação de atividades com as alunas. A fim de avaliar as atividades, $42,9 \%$ das abordagens utilizaram questionários antes e após as atividades programadas e $28,6 \%$ avaliaram através de entrevistas (Tabela 3). Outros métodos empregados foram a inclusão de acampamento ou visitas a empresas locais [Burge 2013] [Gannod 2014], contato direto das estudantes com mulheres profissionais [Burge 2013] e a capacitação e treinamento de professores [Cohoon 2011].

Tabela 3. Metodologias encontradas para o ensino médio

\begin{tabular}{|c|c|}
\hline Metodologia & Estudo \\
\hline Acampamento & [Burge 2013] [Gannod 2014] \\
\hline $\begin{array}{c}\text { Aprendizado baseado em produção (programação, } \\
\text { modelagem, design, prototipação e montagem) }\end{array}$ & $\begin{array}{c}\text { [Burge 2013] [Gannod 2014] [Al-Duwis 2013] } \\
\text { [Rosson 2010] [Alhumoud 2014] }\end{array}$ \\
\hline Avaliação através de questionários & [Burge 2013] [Gannod 2014] [Al-Duwis 2013] \\
\hline Contato direto com profissionais de sucesso na área & [Burge 2013] \\
\hline Capacitação e treinamento para professores & [Cohoon 2011] \\
\hline
\end{tabular}

\subsection{Ensino Fundamental e Médio}

As alunas do ensino fundamental e médio foram público-alvo de cinco trabalhos [Lu and Mead 2012] [Stoeger 2013] [Mikesell and Rinard 2011] [Overdorf and Lang 2011] [Marcu 2010]. As atividades foram planejadas como acampamentos de verão [Marcu 2010], oficinas [Lu and Mead 2012] [Overdorf and Lang 2011] e mentoria online [Stoeger 2013]. Todos trabalharam com grupos exclusivamente de meninas. O trabalho descrito em [Mikesell and Rinard 2011] relata as dificuldades do ponto de vista de uma aluna de Ciência da Computação. Com respeito à metodologia aplicada pelos trabalhos, foram classificadas como mostrado na Tabela 4. As atividades de aprendizagem foram baseadas em produção (programação, modelagem, design, prototipação e montagem); baseadas em jogos educativos [Overdorf and Lang 2011]; lendo livros, assistindo vídeos e participando de discussões sobre a participação de mulheres na tecnologia (STEM) [Stoeger 2013] [Mikesell and Rinard 2011].

\footnotetext{
${ }^{2}$ Podcasts são pequenos áudios gravados e disponibilizados em sites, que podem ser escutados a qualquer momento.
} 
V Congresso Brasileiro de Informática na Educação (CBIE 2016)

Anais dos Workshops do V Congresso Brasileiro de Informática na Educação (CBIE 2016)

Tabela 4. Metodologias encontradas para o ensino fundamental e médio

\begin{tabular}{|c|c|}
\hline Metodologia & Estudo \\
\hline $\begin{array}{c}\text { Aprendizado baseado em produção (programação, } \\
\text { modelagem, design, prototipação e montagem) }\end{array}$ & [Lu and Mead 2012] [Marcu 2010] \\
\hline Aprendizado baseado em jogos & [Overdorf and Lang 2011] \\
\hline Leitura de livros, vídeos e discussões & [Stoeger 2013] [Mikesell and Rinard 2011] \\
\hline Avaliação através de questionários & $\begin{array}{c}\text { [Stoeger 2013] [Overdorf and Lang 2011] } \\
\text { [Marcu 2010] }\end{array}$ \\
\hline Avaliação através do conteúdo produzido & [Marcu 2010] \\
\hline Avaliação através de entrevistas & [Marcu 2010] \\
\hline Contato direto com profissionais de sucesso na área & [Stoeger 2013] \\
\hline
\end{tabular}

Todos os trabalhos utilizaram questionários de avaliação antes e após as atividades programadas, e [Marcu 2010] utilizou, em conjunto, avaliação através do conteúdo produzido e de entrevistas. Outro aspecto empregado na metodologia foi o contato direto das estudantes com mulheres profissionais [Stoeger 2013].

\subsection{Quantidade de Estudantes Envolvidos e Duração das Atividades}

Diversas abordagens aconteceram na forma de acampamentos, geralmente durante o verão, onde os alunos e alunas do ensino médio permanecem em uma universidade ou em um local especial (hotéis, hospedarias) durante um período de tempo que vai de uma semana até três meses. Houve atividades que perduraram por um e dois anos e uma delas foi executada apenas em um dia. Tipicamente, encontram-se mais atividades realizadas em semanas, sendo a duração de uma semana a de maior ocorrência; a quantidade de alunos envolvidos foi em média 30 estudantes.

\subsection{Ferramentas Utilizadas}

As ferramentas utilizadas nos trabalhos estudados foram aplicadas de maneira simples e lúdica em sua maioria. Algumas foram mais frequentes e melhor avaliadas, tanto pelos professores ou responsáveis, como pelas alunas ao final de cada experiência. De acordo com o estudo as ferramentas mais utilizadas foram: Alice $(15,4 \%)$, Lego Mindstorm $(11,5 \%)$, Scratch $(7,7 \%)$ e MIT AppInventor (7,7\%). Destacamos aqui uma oportunidade de pesquisa: é importante produzir um estudo voltado para a avaliação de diferentes ferramentas que realizem comparações entre elas, a fim de permitir a escolha baseada nas características. É necessário conhecer qual ferramenta é indicada para cada faixa etária, sua facilidade de uso, a capacidade de prender a atenção dos alunos, a indicação segundo o nível de conhecimento anterior e outros.

Uma ferramenta pouco convencional é Prototyping on Paper (prototipação em papel) que permite utilizar telas com desenhos ou imagens para simular um aplicativo sendo executado, sendo excelente para introduzir conceitos sobre Interação HumanoComputador (IHC) [Robinson 2014]. 
V Congresso Brasileiro de Informática na Educação (CBIE 2016)

Anais dos Workshops do V Congresso Brasileiro de Informática na Educação (CBIE 2016)

As mulheres sentem-se frequentemente atraídas pela área de Computação quando esta oferece oportunidade de serem criativas, comunicativas e de usarem habilidade sociais. A subárea de IHC possui diversas destas características nas quais as mulheres sentem-se confortáveis, por ser orientada a pessoas, interdisciplinar e colaborativa. A habilidade de projetar aplicações usando prototipação com foco em IHC permite que as meninas comuniquem-se e ajudem as pessoas, o que geralmente as motiva.

A Tabela 5 mostra a frequência de utilização das ferramentas pelos estudos, e a Tabela 6 mostra o tipo de licença de uso de cada ferramenta, classificadas como Gratuita, Paga e Privada. As ferramentas privadas são em geral softwares proprietários desenvolvidos pela própria universidade.

Tabela 5. Frequência das ferramentas

\begin{tabular}{|c|c|}
\hline Ferramenta & Qtde \\
\hline Alice & 4 \\
\hline Lego Mindstorm Kit & 3 \\
\hline Mit AppInventor; Scratch & 2 \\
\hline $\begin{array}{c}\text { Curiosity Grid; Grams' House; } \\
\text { PicoBlocks; PicoCricket Kit; Bridge Tools; } \\
\text { Unity 3D; Finch; Cyber Mentor; iRobot } \\
\text { Create; Rhinoceros 3D; Agent Sheets; } \\
\text { Appcelerator Titanium; Iphone Mockup; } \\
\text { Stagecast Creator; Prototyping on Paper }\end{array}$ & 1 \\
\hline
\end{tabular}

Tabela 6. Tipo de licença das ferramentas

\begin{tabular}{|c|c|}
\hline Ferramenta & Licença \\
\hline Finch, iRobot Create, Lego Mindstorm, & Paga \\
PicoCricket Kit, Rhinoceros & \\
\hline Grams House, The Curiosity Grid & Privada \\
(virtual world), WConnect (community) & \\
\hline Agent Sheets, Alice, Appcelerator & Gratuita \\
Titanium, Bridgetools, CyberMentor, & \\
iPhone Mockup Tool, MIT AppInventor, & \\
PicoBlocks, Prototyping on Paper, & \\
Scratch, Stagecast Creator, Unity & \\
& \\
\end{tabular}

\section{Conclusões}

Neste trabalho foram analisados 23 relevantes estudos publicados entre 2010 e 2015 que apresentam estratégias destinadas a incentivar alunas do Ensino Médio e Fundamental a ingressarem nos cursos superiores e carreiras da Ciência da Computação. Entre eles, onze tiveram foco apenas no Ensino Fundamental, seis no Ensino Médio e outros seis aplicados a ambos. Grande parte das experiências descritas foram realizadas durante acampamentos de verão nos Estados Unidos, uma prática comum naquele país. Parece ser interessante e motivadora o uso desta prática, pois promove a imersão total dos estudantes no ambiente e nas experiências que se deseja fomentar.

As oficinas de aprendizagem foram focadas em sua grande maioria em atividades de produção envolvendo programação, modelagem, design, prototipação e montagem. As atividades realizadas através de jogos educativos aparecem com destaque também. As ferramentas mais utilizadas para o ensino foram Alice, Lego Mindstorm, Scratch e MIT AppInventor, gratuitas exceto pelo Lego. Os conteúdos ministrados versaram na sua maioria sobre o ensino de programação, seguido de desenvolvimento de aplicativos, robótica, jogos educativos e palestras. A grande maioria dos estudos foi realizada com grupos somente de meninas. Um dos estudos misto observou que as alunas se sentem bem mais confortáveis quando trabalham apenas com meninas. Recomenda-se levar em consideração esta pesquisa na programação de atividades para o estímulo ao ingresso de meninas na área de Ciência da Computação. Considera-se que o número de iniciativas para o incentivo de alunas a ingressar na Ciência da 
V Congresso Brasileiro de Informática na Educação (CBIE 2016)

Anais dos Workshops do V Congresso Brasileiro de Informática na Educação (CBIE 2016)

Computação no Brasil ainda é reduzido, sendo enfatizada a necessidade de promover novas abordagens que proporcionem maiores e melhores oportunidades para elas.

\section{Referências}

Al-Duwis, M. et al. (2013). "Increasing high school girls awareness of computer science through summer camp”. In: Global Engineering Education Conf., IEEE.

Alhumoud, S. et al. (2014). "Using app inventor and lego mindstorm nxt in a summer camp to attract high school girls to computing fields". In: Global Engin. Education Conf. IEEE.

Bourdieu, P. (2002). A dominação masculina. Ed. 2. Rio de Janeiro: Bertrand Brasil.

Burge, J. E. et al. (2013). "Girls on the go: a CS summer camp to attract and inspire female high school students". In: Proc. of the 44th ACM tech. symp. on CS Education. ACM.

Cohoon, J.; Cohoon, J. M. e Soffa, M. L. (2011). "Focusing high school teachers on attracting diverse students to computer science and engineering". $41^{\text {st }}$ ASEE/IEEE Frontiers in Education Conference (FIE).

Denner, J.1; Werner, L., Ortiz, E. (2012). "Computer games created by middle school girls: Can they be used to measure understanding of computer science concepts?". Computers \& Education, v. 58, n. 1, p. 240-249.

Gannod, G. C. et al. (2014). "Increasing awareness of computer science in high school girls". In: Frontiers in Education Conference (FIE), IEEE. p. 1-8.

Hirsch, L. S. et al. (2013). "A comparison of single and mixed gender engineering enrichment programs for elementary students". In: Frontiers in Education Conference, IEEE.

Hulsey, C.; Pence, T. B., Hodges, L. F. (2014). "Camp CyberGirls: using a virtual world to introduce computing concepts to middle school girls". In: Proceedings of the 45th ACM technical symposium on Computer science education. ACM.

INEP. (2013). Censo da Educação Superior 2013. http://portal.inep.gov.br/web/censoda-educacao-superior, Abril.

Jung, E., Apedoe, X. (2013). "Changing Young Women's Perceptions of CS via Outreach". 18th Annual Conf. on Innovation and Technology in CS Education.

Khoja, S. et al. (2012). "Changing girls' attitudes towards Computer Science”. Journal of Computing Sciences in Colleges, v. 28, n. 1, p. 210-216.

Lu, D. V., Mead, R. (2012). "Introducing estudantes grades 6-12 to expressive robotics". In: Human-Robot Interaction (HRI), 7th ACM/IEEE International Conference.

Marcu, G. et al. (2010). "Design and evaluation of a computer science and engineering course for middle school girls". In: Proc. of the 41st ACM tech. symp. on CS education. ACM.

Mikesell, A., Rinard, G. (2011). "A deficit of women in computer science: a student's perspective". Journal of Computing Sciences in Colleges, v. 26, n. 3. 
V Congresso Brasileiro de Informática na Educação (CBIE 2016)

Anais dos Workshops do V Congresso Brasileiro de Informática na Educação (CBIE 2016)

Murphy, R. et al. (2010). "Survivor buddy and scigirls: Affect, outreach, and questions". In: Proc. of the 5th ACM/IEEE International Conference on Humanrobot interaction. IEEE.

NTI. (2015). Núcleo de Tecnologia da Informação da UFPB.

OECD. (2013). "OECD Skills Outlook 2013: First Results from the Survey of Adult Skills”. OECD Publishing. http://dx.doi.org/10.1787/9789264204256-en, Abril.

Olinto, G. (2011). "A inclusão das mulheres nas carreiras de ciência e tecnologia no Brasil”. Revista Inclusão Social, vol. 5, No. 1.

Overdorf, R., Lang, M. (2011). "Reaching out to aid in retention: empowering undergraduate women". In: Proc. of the 42nd ACM tech. symp. on CSeducation. ACM.

Pereira, C., Meyer, B. (2013). "Informatics education in Europe: institutions, degrees, students, positions, salaries”. Key Data 2008-2012. Informatics Europe.

PNUD. (2015). Programa das Nações Unidas para o Desenvolvimento. http://www.pnud.org.br.

Robinson, A. (2014). "Underrepresented middle school girls: on the path to computer science through paper prototyping". In: Proc. of 45th ACM Computer science education. ACM.

Rosson, M. B. et al. (2010). "Offering Early Success Experiences in Software Construction: Experiences Teaching Dynamic Website Development to High School Girls". In: Conference on Advanced Learning Technologies, IEEE.

Starrett, C. et al. (2015). "Computational Bead Design: A Pilot Summer Camp in Computer Aided Design and 3D Printing for Middle School Girls". In: Proc. of 46th ACM Symp.

Stewart-Gardiner, C. et al. (2013). "Influencing middle school girls to study computer science through educational computer games". Journal of CS in Colleges, v. 28, n. 6.

Stoeger, H. et al. (2013). "The effectiveness of a one-year online mentoring program for girls in STEM". Computers \& Education, v. 69, p. 408-418.

Webb, D. C.; Repenning, A., Koh, K. H. (2012). "Toward an emergent theory of broadening participation in CS education". In: Proc. of the 43rd ACM Tech. Symposium. ACM.

Webb, H. C. (2011). "Injecting computational thinking into career explorations for middle school girls". In: Visual Languages and Human-Centric Computing (VL/HCC), IEEE Symposium on. IEEE, 2011 . p. 237-238.

Webb, H., Rosson, M. B. (2013). "Using scaffolded examples to teach computational thinking concepts". In: Proc. of the 44th ACM technical symposium on CS education. ACM.

Wellington, C. et al. (2010). "Work in progress-podcasts for recruiting and retaining female computer science majors". In: Frontiers In Education Conference-Global Engineering: Knowledge Without Borders, 2010. 37th Annual. IEEE. 